\title{
Diseño de rúbricas para la evaluación de competencias transversales. Aplicación a Trabajos Fin de Grado en ingeniería
}

\author{
Isabel Martón a , Sergio Gallardo ${ }^{\mathrm{b}}$, José Felipe Villanueva ${ }^{\mathrm{c}}$ y Jose Ordónez ${ }^{\mathrm{d}}$ \\ ${ }^{a}$ Dpto. de Estadística e Investigación Operativa Aplicadas y Calidad, Universitat Politècnica de \\ València, Spain, email: ismarllu@upvnet.upv.es; ${ }^{\circ}$ Dpto. de Ingeniería Química y Nuclear, Universitat

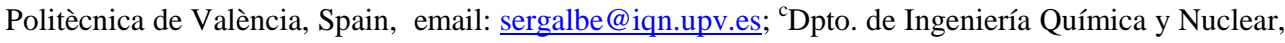 \\ Universitat Politècnica de València, Spain, email: jovillo0@upvnet.upv.es; ${ }^{\mathrm{d} D p t o . ~ d e ~ I n g e n i e r i ́ a ~}$ \\ Química y Nuclear, Universitat Politècnica de València, Spain, email: joorro1@etsii.upv.es
}

\begin{abstract}
In the Context of the European Higher Education Area (EHEA) it is necessary to guarantee that at the end of the degree the different competences have been achived, the specific skills of the subjects studied of each degree and the acquisition of another skills, so-called transversal competences.

In this context, the institutional project of Transversal Competence of the Universitat Politècnica de València (TC-UPV) carries out a classification in 13 competences, worked through three different ways (degree subjects, Bachelor's Thesis (BT)/Master's Thesis (MT) and extracurricular activities) and in different mastery levels.

BT and MT do not have a methodology that systematizes the identification, analysis and the assessment of the competences, among other reasons, for the wide spectrum of subjects studied. In this latter case, the tutor shall be responsible for performing this work.

In order to detect the main problems that students face in the development of their Bachelor's Thesis has been performed the present work. After the tutoring experience gained and through the analysis of different surveys outcomes, it was concluded that effort should be reinforced in the following transversal competences: time management, effective communication, analysis and practical thinking. So, the department has designed specific rubrics of these competences.
\end{abstract}

Keywords: Bachelor's thesis, transversal competencies, assessment, indicators.

\section{Resumen}

En el contexto del Espacio Europeo de Educación Superior es necesario garantizar que a la finalización del grado se han adquirido diferentes competencias, tanto específicas como transversales. 
El proyecto institucional Competencias Transversales de la Universitat Politècnica de València realiza una clasificación de estas, que se trabajan mediante tres vías diferentes (entre ellas los Trabajos Fin de Grado) y en diferentes niveles de dominio. Sin embargo, en TFG no se dispone de una metodología que sistematice la identificación, análisis y evaluación de las competencias, entre otras razones, por el amplio espectro de temas que se estudian, siendo en este caso el tutor el responsable de realizar esta labor.

En este trabajo, se ha elaborado un cuestionario para detectar cuáles fueron los principales problemas durante el desarrollo del TFG por parte de un grupo de alumnos. Tras la experiencia adquirida en la tutorización y mediante el análisis de los resultados del cuestionario, se concluyó que se debía reforzar el trabajo en las siguientes competencias: gestión del tiempo, comunicación efectiva, análisis y pensamiento práctico. Con el objetivo de sistematizar el proceso de evaluación, se han diseñado unas rúbricas específicas para estas competencias.

Palabras clave: Trabajo Fin de Grado, Competencias transversales, evaluación, indicadores.

\section{Introducción}

En el contexto del Espacio Europeo de Educación Superior (EEES) es necesario garantizar que a la finalización del grado se han adquirido diferentes competencias, además de las competencias específicas, propias de las asignaturas cursadas, cada titulación también debe garantizar la adquisición de otra serie de competencias denominadas transversales.

Las Escuelas y Facultades, en colaboración con los profesores, asignan a cada asignatura los puntos de control de trabajo y evaluación para diferentes competencias transversales. En el caso de los Trabajos Fin de Grado (TFG) cada Facultad y Escuela tiene desarrollado su propio programa para el TFG, en el que se especifican las características que debe reunir en cada titulación y sus criterios de evaluación. Sin embargo, en este caso no se dispone de una metodología que sistematice la identificación, análisis y evaluación de las competencias transversales, entre otras razones, por el amplio espectro de temas que se estudian, siendo en este caso el tutor el responsable de realizar esta labor teniendo en consideración las competencias transversales y específicas de cada titulación. Es por ello por lo que en los últimos años se han desarrollado algunos trabajos enfocados a la sistematización, análisis y evaluación de estas competencias transversales en los TFGs.

En (Rullán, 2010) se ha presentado una evaluación de las competencias transversales en los TFG, analizando las características que definen esta materia y los medios e instrumentos para su evaluación. En (Jaume-i-Capó, 2012) se ha realizado un estudio sobre el tipo y el número de competencias transversales establecidas como evaluables dentro de la materia TFG para 64 Grados de la Universitat Autònoma de Barcelona, agrupándolas por rama de conocimiento y diseñando indicadores para algunas de ellas, así como medios e instrumentos para evaluarlas. En (Reyes-García, 2016) se ha analizado la normativa del

(cc) EY-NC-ND 2018, Universitat Politècnica de València

Congreso IN-RED (2018) 
TFG en el enfoque del EEES, analizando si las tareas de evaluación del TFG están relacionadas con las competencias específicas y transversales de sus títulos, comprobando la modalidad de participación del alumnado en la evaluación del TFG y, además, verificando qué función cumple el feedback que se ofrece al alumnado durante el proceso de esta evaluación. En (Herrero, 2011) se propone un modelo de evaluación de competencias transversales siguiendo un esquema tipo cronológico donde se incluye una fase previa, una fase de desarrollo y una fase de finalización.

En el año 2017, la Universidad de Bilbao publicó una guía (Martínez, 2017) para el desarrollo y evaluación continua de las competencias transversales en el TFG mediante dinámicas colaborativas, fomentando la adquisición de competencias transversales tales como la capacidad de reflexión y crítica, habilidad de la comunicación oral y escrita, capacidad de trabajo en equipo o el manejo adecuado de las fuentes bibliográficas.

En este contexto, el proyecto institucional Competencias Transversales de la Universitat Politècnica de València (CT-UPV) realiza una clasificación en 13 competencias transversales, las cuales pretenden sintetizar un perfil competencial para todos los alumnos egresados de la UPV, garantizando que se cubre el marco de referencia de todas las titulaciones, son las siguientes: comprensión e integración, aplicación y pensamiento práctico, análisis y resolución de problemas innovación, creatividad y emprendimiento, diseño y proyecto, trabajo en equipo y liderazgo, responsabilidad ética, medioambiental y profesional. comunicación efectiva, pensamiento crítico, conocimiento de problemas contemporáneos, aprendizaje permanente, planificación y gestión del tiempo e instrumental específica. Estas competencias se trabajan en tres niveles de dominio: el primero se desarrolla en los cursos $1^{\circ}$ y $2^{\circ}$ del grado 2 , el segundo nivel se desarrolla en los cursos $3^{\circ} \mathrm{y}$ $4^{\circ}$ del grado y el tercer nivel se desarrolla en el máster. Además, para incorporar, evaluar y acreditar la adquisición de las CT UPV se establecen tres vías complementarias entre sí: la vía corresponde a planes de estudio, la vía 2 a TFG/TFM y la vía 3 a actividades extracurriculares. El proyecto de la UPV no ha sistematizado el proceso de la evaluación de las competencias transversales en lo que respecta a los TFGs y TFMs. En este caso son los centros los que, para cada titulación, identifican las competencias que se van a trabajar y desarrollan sus propias rúbricas. Estas rúbricas están enfocadas principalmente para facilitar la tarea de evaluación por parte del tribunal y tutor, pero no son útiles para guiar al alumno en la realización del trabajo de forma eficiente.

La innovación presentada en este trabajo se va a llevar a cabo en la unidad docente de energía nuclear del Departamento de Ingeniería Química y Nuclear de la Escuela Técnica Superior de Ingenieros Industriales, en la cual se imparte docencia en los siguientes grados: Grado de Ingeniería en Tecnologías Industriales, Grado de Ingeniería Química, Grado de Ingeniero de la Energía, Grado de Ingeniería en Organización Industrial, Grado de Ingeniería en Biomedicina y diversos másteres. En los últimos cursos académicos, en la unidad docente se han dirigido más de 40 TFGs, en temáticas centradas fundamentalmente en aplicaciones de energía nuclear, energía renovable y eficiencia energética. 


\section{Objetivos}

Los objetivos del presente trabajo son:

- Determinar los principales problemas que los alumnos han encontrado en la realización de sus TFG en relación con las competencias transversales.

- Diseñar herramientas que guíen al alumno en la adquisición de las competencias más problemáticas en el desarrollo del TFG.

\section{Desarrollo de la innovación}

\subsection{Principales problemas durante el desarrollo del TFG}

A raíz de lo comentado en el apartado anterior, se ha considerado necesario valorar en primer lugar los principales problemas que los alumnos afrontan en el desarrollo de sus respectivos TFGs en relación con las competencias transversales sobre las que serán evaluados en su grado de consecución.

Para ello se ha elaborado un cuestionario para detectar dichos problemas. En dicho cuestionario se plantearon las siguientes dimensiones como relevantes para el estudio:

- Grado de conocimiento del programa de competencias transversales.

- Grado de formación en competencias transversales.

- Importancia de las competencias transversales en la realización del TFG.

- Satisfacción en la consecución de estas en la realización del TFG.

En la Tabla 1 se muestra el cuestionario utilizado, así como la escala manejada para su medida.

La petición, instrucciones y acceso web a este cuestionario fue distribuido vía correo electrónico a la base de datos de estudiantes que habían realizado el TFG en la unidad docente durante los últimos cursos académicos. El cuestionario estuvo abierto durante un periodo de 3 semanas con dos recordatorios (a mitad de periodo y poco antes de finalizar el mismo), además de la petición inicial. Se publicó vía web mediante la herramienta "Forms" de Office365. La población estudiada fue de un total de 43 alumnos de los que respondió el $72 \%$ de los mismos (31 de los 43 alumnos).

(cc) EY-NC-ND 2018, Universitat Politècnica de València

Congreso IN-RED (2018) 
Tabla 1. Esquema cuestionario utilizado

\begin{tabular}{|c|c|}
\hline \multicolumn{2}{|c|}{ Grado de Conocimiento de las Competencias Transversales } \\
\hline $\begin{array}{l}\text { ¿Conoces el programa de competencias transversales de la } \\
\text { UPV? }\end{array}$ & Pregunta Dicotómica \\
\hline $\begin{array}{l}\text { ¿Has sido informado por parte de tu tutor de las competencias } \\
\text { transversales sobre las que serás evaluado en tu TFG? }\end{array}$ & Escala Likert \\
\hline $\begin{array}{l}\text { ¿Has sido informado por parte de la escuela de las } \\
\text { competencias transversales sobre las que serás evaluado en tu } \\
\text { TFG? }\end{array}$ & Escala Likert \\
\hline $\begin{array}{l}\text { Valora las siguientes competencias transversales con relación } \\
\text { a tu grado de conocimiento de estas y su aplicabilidad en tu } \\
\text { TFG }\end{array}$ & $\begin{array}{l}\text { Escala de Calificación } \\
\text { continua (1 a 10) (una } \\
\text { por cada competencia) }\end{array}$ \\
\hline \multicolumn{2}{|c|}{ Formación en Competencias Transversales } \\
\hline $\begin{array}{l}\text { ¿Consideras que estás bien formado para desarrollar las } \\
\text { competencias transversales? }\end{array}$ & Escala Likert \\
\hline $\begin{array}{l}\text { ¿Has recibido formación específica para desarrollar las } \\
\text { siguientes competencias transversales? }\end{array}$ & $\begin{array}{l}\text { Escala Likert (una por } \\
\text { cada competencia) }\end{array}$ \\
\hline $\begin{array}{l}\text { ¿Has recibido formación y has trabajado las competencias } \\
\text { transversales dentro de otras asignaturas? }\end{array}$ & $\begin{array}{l}\text { Escala Likert (una por } \\
\text { cada competencia) }\end{array}$ \\
\hline \multicolumn{2}{|c|}{ Importancia de las competencias transversales en la realización del TFG } \\
\hline $\begin{array}{l}\text { Valora las siguientes competencias transversales con relación } \\
\text { a su importancia para la consecución del TFG }\end{array}$ & $\begin{array}{l}\text { Escala de Calificación } \\
\text { continua (1 a 10) (una } \\
\text { por cada competencia) }\end{array}$ \\
\hline \multicolumn{2}{|c|}{ Satisfacción en la consecución de estas en la realización del TFG } \\
\hline $\begin{array}{l}\text { Valora el grado de consecución de las siguientes competencias } \\
\text { transversales }\end{array}$ & $\begin{array}{l}\text { Escala de Calificación } \\
\text { continua (1 a 10) (una } \\
\text { por cada competencia) }\end{array}$ \\
\hline $\begin{array}{l}\text { Valora el grado de información de las siguientes competencias } \\
\text { transversales }\end{array}$ & $\begin{array}{l}\text { Escala de Calificación } \\
\text { continua (1 a 10) (una } \\
\text { por cada competencia) }\end{array}$ \\
\hline $\begin{array}{l}\text { Valora el grado de formación de las siguientes competencias } \\
\text { transversales }\end{array}$ & $\begin{array}{l}\text { Escala de Calificación } \\
\text { continua (1 a 10) (una } \\
\text { por cada competencia) }\end{array}$ \\
\hline & Impresiones Generales \\
\hline $\begin{array}{l}\text { Comenta tus impresiones generales y aquellos puntos de } \\
\text { mejora }\end{array}$ & Respuesta abierta \\
\hline
\end{tabular}


Del análisis de estos resultados previos se extrajeron las primeras conclusiones

- El grado de conocimiento en competencias transversales ha aumentado con el tiempo. Esto no se podía deducir directamente del cuestionario, al que se le tuvo que incorporar datos referentes a la fecha de lectura del TFG.

- El grado de información a los alumnos por parte de las escuelas y tutores es muy limitado, comentando la existencia de éstas y que será evaluado en el mejor de los casos.

- Una gran mayoría evidencia falta de formación específica, aunque si ha recibido cierta formación dentro de otras asignaturas, aunque desde un punto de vista muy general.

- En general son conscientes de la importancia de éstas, aunque alguno no sea de aplicación en sus respectivos TFG

- Pese a la falta de información y formación, se observa que se encuentran medianamente satisfechos con el trabajo realizado en la mayoría de las competencias.

- Particularmente se muestran insatisfechos con la información, formación y desarrollo de las siguientes competencias:

o Análisis y pensamiento práctico.

o Gestión del tiempo.

o Comunicación efectiva.

Al respecto de esto último, reseñar que los TFGs son trabajos de investigación que el estudiante realiza de forma autónoma donde se tiene cierto grado de libertad, tanto en la temática como en los contenidos. Estos TFGs están enfocados para preparar al alumno para la vida profesional, por tanto, un aspecto que resulta importante es la contextualización del tema, lo cual implica un análisis y aplicación práctica del problema que se plantea, además de, una reflexión y actitud crítica. Estos trabajos se asignan al último curso del grado, donde el estudiante se enfrenta al problema de la limitación de tiempo teniendo que ser capaz de ordenar, destruir tiempos, tareas y recursos para alcanzar los resultados de una forma eficiente. En cuanto a la evaluación de estos trabajos, se realiza a través de un documento escrito y de una presentación oral, los cuales deben de ser redactados utilizando expresión clara y precisa y con un formato y estructura de contenidos definidos. Tradicionalmente, la evaluación de estos TFGs se llevaba a cabo bajo criterios de evaluación no explícitos y suelen estar basados en la experiencia del tribunal.

La importancia de estas competencias condujo a la necesidad de incorporar algún tipo de herramienta específica a las mismas que ayudase al alumno y al tutor a desarrollarlas, en especial en lo referente al TFG.

En aras de optimizar el proceso y que fuese lo más autónomo posible se ha propuesto la evaluación de las competencias transversales a partir de rúbricas las cuales están diseñadas de acuerdo con criterios de evaluación, cuantificados mediante indicadores. La rúbrica permite detallar los criterios específicos que se tendrán en cuenta a la hora de evaluar al

(cc) EY-NC-ND 2018, Universitat Politècnica de València

Congreso IN-RED (2018) 
alumno, hacer más fácil el proceso de evaluación al profesor, definir que se espera del alumno en la realización del trabajo, reduce la sensación de ambigüedad y además permite ordenar los conocimientos para realizar una evaluación equitativa. En este contexto, en (Moreno, 2013) se han diseñado varias rúbricas para los TFG en estudios de ingeniería concretamente para las competencias transversales: identificar, formular y resolver problemas propios del ámbito de la ingeniería, comunicación de manera efectiva, y el uso de técnicas y herramientas de ingeniería necesarias en la práctica profesional.

A continuación, se explica el diseño de las rúbricas propuestas.

\subsection{Diseño de rúbricas específicas}

A continuación, se presentan una sería de rúbricas basadas en los indicadores para nivel 2 del proyecto institucional de la Universitat Politècnica de València, adaptadas para su consecución en el desarrollo de un TFG.

Estas rúbricas buscan guiar al alumno en la mejor estrategia para su desarrollo y formación al mismo tiempo facilitando, en lo posible, su incorporación al bagaje propio, pero siendo suficientemente abiertas en función de la singularidad del TFG. Así mismo, también permite al tutor ayudar al alumno facilitando su seguimiento y marcando los puntos importantes a tener en cuenta.

En todos los casos se ha optado por una rúbrica con escala Likert de 4 niveles. Entre los investigadores no hay un consenso claro al respecto. Probablemente el ítem más utilizado sea el de 5 niveles, pero también se utilizan de 4, 7, o 10. Lo que se sabe es que añadir niveles redunda en unas valoraciones más diversas. La más utilizada es la de 5 puntos, pero en estos casos los encuestados suelen evitar las 2 opciones extremas, obteniendo muy poca variación (es el conocido como central tendency bias), por lo que para obtener resultados más claros se ha optado por una escala de 4 niveles.

\subsubsection{Gestión del tiempo}

Esta competencia, en lo que respecta a los TFGs, hace referencia la planificación adecuada de las diferentes actividades en el tiempo disponible, alcanzando los objetivos fijados.

La gestión del tiempo es uno de los aspectos que la mayoría de los estudiantes han detectado como más conflictivo en la realización de los TFGs. Disponer de un buen índice, donde estén definidos los contenidos y objetivos principales puede ayudar a la realización de un buen plan de trabajo, donde estén definidas las duraciones de las actividades y su jerarquización según su importancia. Por tanto, se concretan los siguientes indicadores para medir esta competencia:

- Definir el índice, contenidos y objetivos.

- Determinar las actividades y plazos a desarrollar para que sean acorde con los objetivos.

- Cumplimiento de los objetivos.

- Analizar el desarrollo de las actividades.

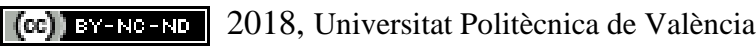

Congreso In-Red (2018) 
En la Tabla 2 se muestra la rúbrica específica propuesta para la Gestión del Tiempo en un TFG.

Tabla 2. Rúbrica Competencia Transversal Gestión del Tiempo para TFG

\begin{tabular}{|c|c|c|c|c|}
\hline & \multicolumn{4}{|c|}{ Gestión del tiempo TFG } \\
\hline Indicadores & Excelente & Notable & Suficiente & Insuficiente \\
\hline $\begin{array}{lr}\text { Definir } & \text { índice, } \\
\text { contenidos } & \mathrm{y} \\
\text { objetivos } & \end{array}$ & $\begin{array}{l}\text { Plantea una tabla } \\
\text { de contenidos } \\
\text { desde el inicio } \\
\text { acorde con los } \\
\text { objetivos } \\
\text { definidos }\end{array}$ & $\begin{array}{lr}\begin{array}{l}\text { Plantea } \\
\text { tabla una } \\
\text { contenidos }\end{array} \\
\text { durante de } \\
\text { realización del } \\
\text { trabajo siendo } \\
\text { acorde con los } \\
\text { objetivos } \\
\text { definido }\end{array}$ & $\begin{array}{l}\text { Plantea una tabla } \\
\text { de contenidos } \\
\text { durante la } \\
\text { realización del } \\
\text { trabajo no siendo } \\
\text { acorde con los } \\
\text { objetivos } \\
\text { definido }\end{array}$ & $\begin{array}{l}\text { No plantea una } \\
\text { tabla de } \\
\text { contenidos } \\
\text { desde el } \\
\text { principio } \\
\text { acorde con los } \\
\text { objetivos } \\
\text { definidos }\end{array}$ \\
\hline $\begin{array}{lr}\text { Determinar } & \text { las } \\
\text { actividades } & \text { a } \\
\text { desarrollar a medio } \\
\text { plazo, de forma } \\
\text { acorde con los } \\
\text { contenidos } \\
\text { objetivos }\end{array}$ & $\begin{array}{lr}\text { Especifica } & \text { todas } \\
\text { las actividades a } \\
\text { realizar y las } \\
\text { relaciona con el } \\
\text { índice r de } \\
\begin{array}{lr}\text { contenidos } \\
\text { objetivos }\end{array}\end{array}$ & $\begin{array}{lr}\text { Especifica } & \text { la } \\
\text { mayoría } & \text { de } \\
\text { actividades } & \text { a } \\
\text { realizar Prioriza } \\
\text { en función de su } \\
\text { urgencia las } \\
\text { actividades a } \\
\text { realizar }\end{array}$ & $\begin{array}{l}\text { Especifica } \\
\text { algunas } \\
\text { actividades. }\end{array}$ & $\begin{array}{l}\text { No especifica } \\
\text { actividades a } \\
\text { desarrollar. }\end{array}$ \\
\hline $\begin{array}{l}\text { Planificación del } \\
\text { trabajo }\end{array}$ & \begin{tabular}{lr}
\multicolumn{2}{l}{ Planifica } \\
\multicolumn{2}{l}{ adecuadamente } \\
todas las \\
actividades & para \\
alcanzar los \\
objetivos, \\
asignando \\
tiempos a \\
actividades \\
prioridades
\end{tabular} & $\begin{array}{lr}\text { Planifica } & \text { las } \\
\text { actividades } & \text { para } \\
\text { alcanzar } & \text { los } \\
\text { objetivos } & \\
\text { asignando } & \\
\text { tiempos } & \text { sin } \\
\text { priorizar. } & \end{array}$ & $\begin{array}{lr}\text { Planifica } & \text { de } \\
\text { forma errónea } \\
\text { algunas de las } \\
\text { actividades para } \\
\text { alcanzar los } \\
\text { objetivos. No se } \\
\text { asignan tiempos } \\
\text { a las actividades } \\
\text { ni prioridades }\end{array}$ & $\begin{array}{l}\text { No planifican } \\
\text { las actividades } \\
\text { para alcanzar } \\
\text { los objetivos }\end{array}$ \\
\hline $\begin{array}{ll}\text { Analizar } & \text { el } \\
\text { desarrollo de las } \\
\text { actividades. }\end{array}$ & $\begin{array}{lr}\text { Analiza } & \text { el } \\
\text { avance de las } \\
\text { actividades } & y \\
\text { propone } & \text { mejoras } \\
\text { durante ra } & \text { la } \\
\text { realización del } \\
\text { trabajo. }\end{array}$ & $\begin{array}{lr}\text { Analiza } & \text { el } \\
\text { avance de las } \\
\text { actividades de } \\
\text { forma } \\
\text { superficial, } \\
\text { proponiendo } \\
\text { algunas mejoras }\end{array}$ & $\begin{array}{ll}\text { Realiza } & \text { un } \\
\text { análisis } & \\
\text { inadecuado del } \\
\begin{array}{ll}\text { avance de las } \\
\text { actividades }\end{array}\end{array}$ & $\begin{array}{l}\text { No realiza } \\
\text { ningún } \\
\text { seguimiento y } \\
\text { análisis. }\end{array}$ \\
\hline
\end{tabular}

(c)) EY-NC-ND 2018, Universitat Politècnica de València 


\subsubsection{Comunicación Efectiva}

La comunicación efectiva, en lo que respecta a los TFGs tiene dos dimensiones, ya que los estudiantes deben de realizar un documento escrito y defender oralmente su trabajo frente a un tribunal. El alumno en los dos casos debe de comunicarse de forma clara y precisa utilizando un buen lenguaje y siguiendo una estructura y organización equilibrada. En lo que respecta a la comunicación oral, se añaden aspectos tales como la buena comunicación no verbal, el uso de medios de apoyo y el ajustarse al tiempo establecido en la presentación. Por tanto se concretan los siguientes indicadores:

- Escritura y gramática.

- Estructura y organización.

- Contenidos de la exposición oral.

- $\quad$ Ajuste al tiempo de la exposición.

- Lenguaje no verbal.

- $\quad$ Respuestas al tribunal.

En la Tabla 3 se muestra la rúbrica específica propuesta para la Comunicación Efectiva de un TFG.

Tabla 3. Rúbrica Competencia Transversal Comunicación Efectiva para TFG

\begin{tabular}{|c|c|c|c|c|}
\hline & \multicolumn{4}{|c|}{ Comunicación efectiva TFG } \\
\hline Indicadores & Excelente & Notable & Suficiente & Insuficiente \\
\hline $\begin{array}{ll}\begin{array}{l}\text { Escritura } \\
\text { gramática }\end{array} & \mathrm{y} \\
\end{array}$ & $\begin{array}{l}\text { Se entiende bien } \\
\text { lo que está } \\
\text { escrito. Utiliza } \\
\text { bien el idioma, } \\
\text { el lenguaje } \\
\text { científico y su } \\
\text { gramática. }\end{array}$ & $\begin{array}{l}\text { Necesita } \\
\text { mejorarse }\end{array}$ & $\begin{array}{l}\text { Estructura confusa } \\
\text { y difícil de leer }\end{array}$ & Ilegible \\
\hline $\begin{array}{ll}\text { Estructura } & y \\
\text { organización } & \end{array}$ & $\begin{array}{l}\text { Los contenidos } \\
\text { están } \\
\text { equilibrados y } \\
\text { bien } \\
\text { organizados. } \\
\text { Contiene los } \\
\text { apartados } \\
\text { establecidos. Se } \\
\text { centra en las } \\
\text { ideas más } \\
\text { importantes. }\end{array}$ & $\begin{array}{l}\text { La estructura } \\
\text { esta pasable, } \\
\text { falta algún } \\
\text { apartado } \\
\text { establecido. }\end{array}$ & $\begin{array}{l}\text { Los contenidos y } \\
\text { organización están } \\
\text { descompensados. } \\
\text { Faltan varios } \\
\text { apartados } \\
\text { establecidos. }\end{array}$ & $\begin{array}{l}\text { Desestructurado } \\
\text { y con } \\
\text { información } \\
\text { repetida }\end{array}$ \\
\hline $\begin{array}{l}\text { Contenidos de } \\
\text { la exposición } \\
\text { oral }\end{array}$ & $\begin{array}{l}\text { Se seleccionan } \\
\text { adecuadamente } \\
\text { los contenidos y } \\
\text { se expone de }\end{array}$ & $\begin{array}{l}\text { Se seleccionan } \\
\text { bien los } \\
\text { contenidos pero } \\
\text { se explican de }\end{array}$ & \begin{tabular}{lr}
\multicolumn{2}{l}{ No se seleccionan } \\
bien & los \\
contenidos. & La \\
presentación & es
\end{tabular} & $\begin{array}{l}\text { La presentación } \\
\text { no incluye los } \\
\text { aspectos } \\
\text { fundamentales. }\end{array}$ \\
\hline
\end{tabular}

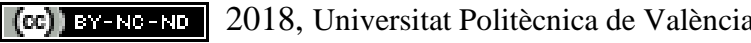

Congreso In-Red (2018) 


\begin{tabular}{|c|c|c|c|c|}
\hline & 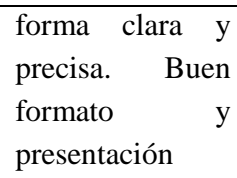 & forma confusa & difícil de seguir & $\begin{array}{l}\text { Mala } \\
\text { presentación } \\
\text { formato }\end{array}$ \\
\hline $\begin{array}{ll}\text { Ajuste } & \text { al } \\
\text { tiempo de } & \text { la } \\
\text { exposición } & \end{array}$ & $\begin{array}{l}\text { Se ajusta } \\
\text { correctamente al } \\
\text { tiempo de la } \\
\text { presentación. } \\
\text { Expone a un } \\
\text { ritmo constante, } \\
\text { notándose que la } \\
\text { presentación está } \\
\text { preparada y } \\
\text { ensayada. }\end{array}$ & $\begin{array}{l}\text { Se ajusta al } \\
\text { tiempo de la } \\
\text { presentación, } \\
\text { aunque en la } \\
\text { parte final se } \\
\text { acelera el } \\
\text { ritmo. }\end{array}$ & $\begin{array}{lr}\text { Se sobrepasa } & \text { el } \\
\text { tiempo } & \text { de } \\
\text { exposición, aunque } \\
\text { el tribunal no } \\
\text { interrumpe ra } \\
\text { exposición }\end{array}$ & $\begin{array}{l}\text { El tribunal debe } \\
\text { de interrumpir la } \\
\text { exposición, no se } \\
\text { cumplen los } \\
\text { tiempos } \\
\text { acordados. }\end{array}$ \\
\hline $\begin{array}{ll}\text { Lenguaje } & \text { no } \\
\text { verbal } & \end{array}$ & $\begin{array}{l}\text { Presentación con } \\
\text { buen tono de } \\
\text { voz, el alumno } \\
\text { está tranquilo, } \\
\text { enfatiza en lo } \\
\text { más importante. }\end{array}$ & $\begin{array}{l}\text { Presentación } \\
\text { con buen tono } \\
\text { de voz aunque } \\
\text { el alumno se } \\
\text { nota } \\
\text { intranquilo. }\end{array}$ & $\begin{array}{l}\text { Presentación con } \\
\text { mal tono de voz, } \\
\text { demostrando } \\
\text { intranquilidad e } \\
\text { inseguridad. }\end{array}$ & $\begin{array}{l}\text { Exposición } \\
\text { inaudible, } \\
\text { monótona. }\end{array}$ \\
\hline $\begin{array}{l}\text { Respuestas al } \\
\text { tribunal }\end{array}$ & $\begin{array}{l}\text { Responde } \\
\text { correctamente a } \\
\text { las preguntas } \\
\text { planteadas, de } \\
\text { forma concisa, } \\
\text { notándose que se } \\
\text { conoce la } \\
\text { materia. }\end{array}$ & $\begin{array}{l}\text { Responde de } \\
\text { forma errónea } \\
\text { las preguntes } \\
\text { planteadas. }\end{array}$ & $\begin{array}{llr}\text { Responde a las } \\
\text { preguntas } & & \text { sin } \\
\text { seguridad y } & \text { de } \\
\text { forma dubitativa. }\end{array}$ & $\begin{array}{lr}\text { No } & \text { sabe } \\
\text { responder a las } \\
\text { preguntas } & \\
\text { planteadas. } & \end{array}$ \\
\hline
\end{tabular}

\subsubsection{Análisis y pensamiento práctico}

Con esta competencia se busca que el alumno aplique los conocimientos teóricos y establezca los procesos a seguir para alcanzar sus objetivos, analice e interprete los datos para extraer conclusiones, en especial en lo referente a su TFG. Para ello se estableen los siguiente s indicadores:

- Obtener la información necesaria para abordar las situaciones.

- Evaluar la calidad de la información de que dispone para su aplicación.

- Realizar la contextualización del problema.

- Establecer la metodología y definir datos de entrada y salida.

- Elaborar un plan coherente para resolver las situaciones que se le planteen.

En la Tabla 4 se muestra la rúbrica específica propuesta para el Análisis y Pensamiento Práctico en un TFG. 
Tabla 4. Rúbrica Competencia Transversal Análisis y Pensamiento Práctico para TFG

\begin{tabular}{|c|c|c|c|c|}
\hline \multirow[b]{2}{*}{ Indicadores } & \multicolumn{4}{|c|}{ Análisis y Pensamiento Práctico TFG } \\
\hline & Excelente & Notable & Suficiente & Insuficiente \\
\hline $\begin{array}{lr}\text { Obtener } & \text { la } \\
\text { información } & \\
\text { necesaria } & \text { para } \\
\text { abordar } & \text { las } \\
\text { situaciones } & \end{array}$ & $\begin{array}{l}\text { Hay una } \\
\text { búsqueda } \\
\text { exhaustiva de } \\
\text { información y se } \\
\text { comenta }\end{array}$ & $\begin{array}{l}\text { Hay una } \\
\text { búsqueda } \\
\text { exhaustiva de } \\
\text { información, } \\
\begin{array}{ll}\text { pero no se } \\
\text { comenta }\end{array}\end{array}$ & $\begin{array}{lr}\text { Se } & \text { busca } \\
\text { información de } \\
\text { pocas fuentes }\end{array}$ & $\begin{array}{l}\text { No hay búsqueda } \\
\text { de información }\end{array}$ \\
\hline \begin{tabular}{lr} 
Evaluar & la \\
calidad de la \\
información de \\
que dispone para \\
\multicolumn{2}{l}{ su aplicación. }
\end{tabular} & $\begin{array}{l}\text { Toda la } \\
\text { información es } \\
\text { de fuentes fiables } \\
\text { y contrastables }\end{array}$ & $\begin{array}{l}\text { La mayor parte } \\
\text { de la información } \\
\text { es de fuentes } \\
\text { fiables }\end{array}$ & $\begin{array}{l}\text { Parte de la } \\
\text { información es de } \\
\text { fuentes fiables }\end{array}$ & $\begin{array}{l}\text { La información } \\
\text { es de baja } \\
\text { calidad }\end{array}$ \\
\hline $\begin{array}{l}\text { Realizar la } \\
\text { contextualización } \\
\text { del problema que } \\
\text { se plantea }\end{array}$ & $\begin{array}{l}\text { Realiza una } \\
\text { contextualización } \\
\text { del problema, } \\
\text { relacionando un } \\
\text { análisis del } \\
\text { estado actual del } \\
\text { tema. }\end{array}$ & \begin{tabular}{lr} 
Realiza & una \\
\multicolumn{2}{l}{ contextualización } \\
pobre & del \\
problema, sin \\
relacionarlo con \\
el estado actual \\
del tema.
\end{tabular} & $\begin{array}{l}\text { Realiza una mala } \\
\text { contextualización } \\
\text { del tema. }\end{array}$ & $\begin{array}{l}\text { No realiza una } \\
\text { contextualización } \\
\text { del tema. }\end{array}$ \\
\hline $\begin{array}{l}\text { Establecer la } \\
\text { metodología y } \\
\text { definir datos de } \\
\text { entrada y salida }\end{array}$ & $\begin{array}{l}\text { Elige y aplica } \\
\text { bien la } \\
\text { metodología y } \\
\text { define } \\
\text { adecuadamente } \\
\text { los datos de } \\
\text { entrada y salida } \\
\text { del problema. }\end{array}$ & $\begin{array}{l}\text { Elige } \\
\text { adecuadamente } \\
\text { la metodología, } \\
\text { pero la aplica de } \\
\text { forma parcial. } \\
\text {,Los datos de } \\
\text { entrada y salida } \\
\text { están bien } \\
\text { definidos. }\end{array}$ & $\begin{array}{l}\text { Elige bien la } \\
\text { metodología, pero } \\
\text { no la aplica } \\
\text { adecuadamente. } \\
\text { Los datos de } \\
\text { entrada y salida } \\
\text { están r mal } \\
\text { definidos. }\end{array}$ & $\begin{array}{l}\text { No realiza una } \\
\text { buena elección de } \\
\text { la metodología, } \\
\text { ni de los datos de } \\
\text { entrada y salida. }\end{array}$ \\
\hline $\begin{array}{l}\text { Elaborar un plan } \\
\text { coherente para } \\
\text { resolver las } \\
\text { situaciones que } \\
\text { se le planteen. }\end{array}$ & $\begin{array}{l}\text { Elabora } \text { un plan } \\
\text { de trabajo } \\
\text { coherente con } \\
\text { actualizaciones }\end{array}$ & $\begin{array}{l}\text { Elabora un plan } \\
\text { de trabajo } \\
\text { coherente }\end{array}$ & $\begin{array}{ll}\text { Elabora } & \text { un } \\
\text { esquema asico } & \text { de } \\
\text { trabajo } & \\
\end{array}$ & \begin{tabular}{lr} 
No & \multicolumn{2}{c}{ elabora } \\
ningún plan de \\
trabajo
\end{tabular} \\
\hline
\end{tabular}




\section{Conclusiones}

En el presente trabajo se han diseñado las rúbricas para tres competencias transversales para la realización de los TFGs, elegidas tras analizar los datos del cuestionario previo enviado a los alumnos. Estas rúbricas están diseñadas con el objetivo de que sirvan, aparte de para facilitar el proceso de evaluación de los tutores y tribunal, sirvan al alumnado de guía y de ayuda para reducir algunas dificultades durante el proceso de realización de sus TFGs. Estas rúbricas actualmente se encuentran en fase de diseño y desarrollo y no han podido ser verificadas, no pudiendo medir su eficiencia. Esta tarea de verificación se pretende realizar en el futuro con la distribución de estas rúbricas a los alumnos que previamente realizaron el cuestionario con el objetivo de poseer el punto de vista del alumnado y verificar la idoneidad de estas.

\section{Agradecimientos}

Este trabajo se ha realizado en el marco del proyecto Proyecto de Innovación y Mejora Educativa, PIME Curso 2017-2018 "Coordinación de competencias transversales en asignaturas de ámbito nuclear en el Grado de Ingeniero de la Energía”, Referencia B07, del Vicerrectorado de Estudios, Calidad y Acreditación de la Universitat Politècnica de València.

\section{Referencias}

RULLAN M., FERNÁNDEZ M., ESTAPÉ G., MARQUEZ D. (2010) “The assessment of generic competences in the final year thesis. a preliminary study on the need and opportunity to establish means and tools by fields of knowledge" Revista de Docencia Universitaria, Vol.8 (n.1) 74-100 ISSN:1887- 4592

JAUME I CAPÓ, A., GUERRERO, C., MIRO, J., EGEA A., "Elaboración de una rúbrica para la evaluación TFG y TFM de informática en la Universitat de les Illes Balears”. Actas Simposio-Taller JENUI 2012, Ciudad Real, 10-13 de julio 2012 I.S.B.N. 10: 84-695-39418

REYES-GARCIA C. DÍAZ-MEGOLLA, A.(2017) “¿se adecúa la normativa del Trabajo Fin de Grado al enfoque de evaluación del Espacio Europeo de Educación Superior? ” Rev. complut. educ. 28(4) 2017: 1285-1302, ISSN: 1988-2793

HERRERO, R., FERRER M.A., SOLANO J.P., CALDERÓN A.A., BUSQUIER S. “ Evaluación de competencias transversales en los trabajos de fin de grado”. Actas del Congreso Internacional de Innovación docente, Universidad Politécnica de Cartagena, 6-8 de Julio 2011.

MORENO, V., CARPINTERO, G., HERNANDEZ, D. (2013). "Dos casos del uso de rúbricas para la evaluación de Trabajos de Final de Grado”. III Jornadas de Innovación Educativa en Ingeniería Telemática Universitat Pompeu Fabra de Barcelona

(cc) EY-NC-ND 2018, Universitat Politècnica de València

Congreso IN-RED (2018) 
MARTÍNEZ O., MIRANDA J., SARRIONANDIA

F., TXURRUCA I. (2017). "Guía para el desarrollo y evaluación continua de las competencias transversales en el Trabajo Fin de Grado mediante dinámicas colaborativas", Servicio Editorial Universidad del País Vasco. ISBN: 978-84-9082-721-5 